\title{
The role of commitment in producing misinformation effects in eyewitness memory
}

\author{
THOMAS A. SCHREIBER and SUSAN D. SERGENT \\ University of Kansas, Lawrence, Kansas
}

\begin{abstract}
We conducted three experiments exploring conditions in which misleading postevent information interferes with people's ability to remember details about an event they witnessed. The key condition included in each experiment was the misled-plus-commit condition. After viewing slides depicting a crime, subjects in this condition read a narrative that contained misinformation. Following the narrative, they completed an interpolated recognition test that induced them to select the misinformation. Assessment of memory for the slides using a final, modified recognition test indicated that performance in the misled-plus-commit condition was most frequently near chance, whereas performance in the control condition was far above chance. This result was obtained on four separate occasions and indicates that prior retrieval of misinformation impairs memory. Another important finding was that the deleterious effect of passively reading about misinformation in a narrative is not as great as the effect of reading about it and then selecting it on an interpolated test. Actively retrieving misinformation seems to cause particularly deleterious effects. Our conclusion is that the findings are compatible with the retrieval blocking hypothesis, which assumes that repeated retrieval of misinformation blocks access to the witnessed information.
\end{abstract}

After people witness a crime, they may be exposed to misleading information about details associated with it. For example, having seen a robbery in which a man held a "hammer," a person may be exposed to misinformation indicating that the man held a "screwdriver." How does exposure to such misinformation affect people's ability to remember what they saw? To answer this question in laboratory studies, subjects typically see slides depicting a crime and, following the slides, they read a narrative containing either misinformation or control statements. Finally, memory for the slides is tested. Often a standard recognition test is used in which the response choices are the original detail and the misinformation (e.g., "hammer" and "screwdriver"). Typically, subjects select the correct item less frequently in the misled condition than in the control condition (see, e.g., Loftus, 1979; Loftus, Miller, \& Burns, 1978), and this finding is called the misinformation effect.

Although the misinformation effect has been obtained many times, its magnitude depends on how memory for the original event is tested (McCloskey \& Zaragoza, 1985).

Portions of this research were reported at the 37 th annual meeting of the Psychonomic Society in Chicago. We would like to thank Henry Roediger III, Stephen Lindsay, Robert Belli, and Maria Zaragoza for their comments on earlier versions of this article. T.A.S. would also like to extend very special thanks to Beth Loftus, Doug Nelson, and Jim Jenkins for their support and encouragement. Correspondence should be addressed to T. A. Schreiber, Department of Psychology, University of Kansas, 426 Fraser Hall, Lawrence, KS 66045 (e-mail: schreibe(a) kuhub.cc.ukans.edu).
The difference between accuracy in the control and misled conditions is greater on standard recognition tests in which the misinformation is a response choice than it is on modified recognition tests in which the misinformation is replaced with a novel item (e.g., "hammer" and "wrench"). McCloskey and Zaragoza explained this finding by assuming that the standard test can be influenced by response biases and social demands, whereas the modified test cannot. Response biases occur when people fail to encode the original information and select the misinformation on the standard test because they are biased by having read about the misinformation in the narrative. Social demands occur when people select the misinformation because they decide to comply with the authority of the experimenter who wrote the narrative even though they can remember the original detail. Neither response biases nor social demands can occur on the modified test because the misinformation is not included as a response choice. McCloskey and Zaragoza suggested that the misinformation effect obtained on standard tests does not reflect memory impairment because the effects of response biases and social demands cannot be eliminated.

Although McCloskey and Zaragoza's (1985) nonimpairment hypothesis can explain the dependence of the misinformation effect on the type of test used, there are other explanations, too. The retrieval blocking hypothesis assumes that retrieving erroneous information can block or interfere with access to correct information (see, e.g., Anderson \& Spellman, 1995; McGeoch, 1942; Nelson, Schreiber, \& McEvoy, 1992; Raaijmakers \& Shiffrin, 
1981; Roediger \& Neely, 1982). When applied to the eyewitness paradigm, the blocking hypothesis would assume that retrieving the misinformation is less likely on the modified test than on the standard test because when the misleading item is removed as a response choice, the most important cue for it is removed (see Chandler, 1991). Consequently, on the modified test, retrieval of the misinformation is less likely and the amount of interference produced is lower than on the standard test.

Both the nonimpairment hypothesis and the blocking hypothesis make the same prediction with respect to the effect of testing procedure, and both could be right because they are not mutually exclusive. Nevertheless, there are findings that the blocking hypothesis can explain that the nonimpairment hypothesis cannot. In an investigation that is very relevant to the present research, Schooler, Foster, and Loftus (1988) obtained the misinformation effect on the modified test when people also completed an interpolated test. The key feature of the interpolated test was that the response choices consisted of two incorrect items. When people selected one of these items, they were less likely to respond correctly on the final, modified test than people in the control condition who did not complete an interpolated test. The nonimpairment hypothesis cannot explain this finding because the modified test is immune to the effects of response biases and social demands. As such, Schooler et al. rejected the nonimpairment hypothesis and concluded instead that actively choosing and "committing" to erroneous information on one test causes interference on a second test. ${ }^{1}$

The blocking hypothesis would explain the findings of Schooler et al. (1988) by assuming that retrieval of misinformation in the presence of a memory cue increases the probability with which that cue will elicit the misinformation in the future (see Raaijmakers \& Shiffrin, 1981). When the association between the test cue and the misinformation is strengthened by prior retrieval, the misinformation may be retrieved repeatedly on a given test trial and this process of repeated retrieval blocks access to the memory of the correct information. Although the blocking hypothesis can explain the effect of committing to misinformation, Belli (1993) argued against this hypothesis on the basis of other findings. Like Schooler et al., subjects in Belli's experiments also completed an interpolated test. However, unlike Schooler et al., Belli found that accuracy on the final, modified test was equivalent in the misled and control conditions.

One important purpose of the present research was to determine why interpolated test manipulations do not always cause interference on modified recognition tests. We first will discuss differences between the procedures used by Schooler et al. (1988) and Belli (1993) that could have caused different outcomes to occur. We then will report three experiments that tested the blocking hypothesis further by exploring conditions in which interpolated tests will impair memory. There are two main reasons why Schooler et al. and Belli may have obtained different outcomes. The first concerns the nature of the interpolated tests. In Schooler et al., subjects were prevented from responding correctly on the interpolated test because both response choices were incorrect. In contrast, Belli did not prevent subjects from responding correctly. In one experiment, subjects completed an interpolated recall test after reading the narrative, and they could respond correctly either by guessing or by remembering the original detail. In both cases subjects could "commit" to the correct item. Committing to the correct item should mask the effect of committing to the misleading item. In fact, Belli's results showed that in the misled condition, the correct item was reported on the interpolated test nearly one third of the time, and the correct item was then selected again on the final test $96 \%$ of the time. Roediger, Jacoby, and McDermott (1996) obtained similar results. When people reported the correct item on an interpolated recall test, they were more likely to report it again on a second recall test than were people who reported misinformation on the interpolated test. Similar to Belli's findings, misinformation effects were reduced or entirely absent on the second test in conditions associated with a higher rate of correct responding on the first test. Both sets of findings are compatible with the blocking hypothesis because prior commitment to the correct item should block access to the misleading item and less interference should be produced. ${ }^{2}$

The second difference between the procedures used by Belli (1993) and Schooler et al. (1988) that could have caused different outcomes to occur concerns the materials. Belli used McCloskey and Zaragoza's (1985) materials, whereas Schooler et al. did not. For reasons that are unclear, obtaining the misinformation effect is difficult with McCloskey and Zaragoza's materials when the modified test is used. In a meta-analysis conducted by Payne, Toglia, and Anastasi (1994), none of the 14 studies that obtained significant misinformation effects on the modified test used these materials. In addition, of the 16 studies that did use them, not a single study obtained significant misinformation effects! As such, the different outcomes obtained by Schooler et al. and Belli may be unrelated to the nature of the interpolated test used. Rather, the different outcomes could simply have been due to the use of different materials.

To address these issues, we conducted three experiments using variations of the procedures used by Schooler et al. (1988) and Belli (1993). Like Belli, we used McCloskey and Zaragoza's (1985) materials in order to eliminate differential effects associated with the use of different materials. Also like Belli, we always included the misledplus-commit condition, in which subjects first read a narrative and then completed an interpolated test. Unlike Belli, we used an interpolated test in which subjects could not commit to the correct information. On this test, the misleading item from the narrative was paired with a novel item (e.g., "screwdriver" and "pliers"). We assumed that including the misleading item would induce people to select it because they had read about it in the narrative immediately beforehand. Most important, the procedure of not including the original item as a response choice en- 
sured that the effect of committing to correct information could not mask the effect of committing to misleading information. As such, the effect of committing to misinformation was isolated from the effect of committing to correct information.

\section{EXPERIMENTS 1 AND 2}

The purpose of Experiments 1 and 2 was to demonstrate that the misinformation effect would occur on the modified test using the misled-plus-commit procedure. The procedure in both experiments was similar, so we will report both together to conserve space.

\section{Method \\ Subjects. In Experiment 1, the subjects were 33 students from the University of Washington, Seattle. In Experiment 2, the subjects were 48 students from the University of South Florida, Tampa. All subjects received course credit.}

Procedure and Materials. Both experiments consisted of four phases. Subjects viewed the slides, read the narrative, completed the interpolated test, and then took the final test. The slides from McCloskey and Zaragoza (1985) were presented at a rate of $6 \mathrm{sec}$. They depict a maintenance man conducting repair work in an office from which he steals some items. In Experiment 1, there were two critical items. We used three different versions of each critical item with each version shown to the same number of subjects. The two critical items and the three versions of each were a tool (hammer, wrench, or screwdriver) and a can of soda (Coke, 7-Up, or Sunkist). In Experiment 2, additional items were included: a jar of coffee (Folger's, Maxwell House, or Nescafe) and a magazine (Glamour, Vogue, or Mademoiselle). Following the slides, subjects completed a filler task, which consisted of rating cartoons for humor in Experiment 1 and answering general knowledge questions in Experiment 2. Subjects then read the narrative containing misinformation about half of the critical items and generic information about the remaining half (e.g., "tool"). Each critical item was used equally often in the misled and control conditions, and each of the three versions was used equally often as the slide item and the misleading item. Subjects completed the interpolated test immediately following the narrative. Some test questions were filler questions and others pertained to the critical items. In the misled-plus-commit condition, the response choices for critical questions consisted of the misleading item paired with a novel distractor (e.g., "screwdriver" and "pliers"). In the control condition, the two choices consisted of generic terms (e.g.. "tool" and "sandwich"). Because interpolated test questions were used in each condition, the only difference between them concerned the nature of the response choices. Following the interpolated test, subjects completed another filler task and they then completed the modified test. Some of the questions on the modified test were new and others were similar to those used on the interpolated test. The two response choices for the critical questions consisted of the correct item paired with a novel distractor (e.g., "hammer" and "wrench"). Subjects were asked to base their answers only on their memory of the slides.

\section{Results and Discussion}

In the control condition of Experiment 1, the probability of correct recognition on the modified test was .75 compared with a probability of .47 in the misled-pluscommit condition. A chi-square test using Yates's correction indicated that the difference between these means was significant $\left(\chi^{2}=5.31\right)$. The same pattern occurred in the second experiment. Mean probability of correct recognition was .67 in the control condition and .52 in the misled-plus-commit condition $[t(47)=2.86$; pooled variance $=.02]$. Finally, in the misled-plus-commit condition of both experiments, the misinformation was selected $89 \%$ of the time on the interpolated test. We assume that commitment to the misinformation on the interpolated test caused the near-chance level of performance on the final test. These results confirm those of Schooler et al. (1988). Large misinformation effects occurred on the modified test when using the misied-plus-commit procedure and McCloskey and Zaragoza's (1985) materials. Prior commitment to misinformation clearly interfered with performance on the final test.

\section{EXPERIMENT 3}

This experiment was designed to determine whether more interference is produced when subjects commit to misinformation than when they only read about misinformation. We included both the misled-plus-commit condition and the misled-only condition, which involved the typical procedure of presenting misinformation only in the narrative. We also varied how strongly the original event was encoded by showing the slides either once or twice. In addition, some subjects were given the modified test, as before, whereas others were given the standard test. According to the blocking hypothesis, the misinformation effect should be larger in the misled-plus-commit condition than in the misled-only condition, regardless of the type of final test. In addition, accuracy on the standard test should be worse than on the modified test because the misinformation should be more accessible when it is presented as a retrieval cue. Finally, recognition accuracy should be higher following two slide presentations than following a single presentation in both the control and misled conditions because the memory of the original information should be more accessible regardless of the type of postevent information presented.

\section{Method}

Design and Subjects. The design was a $2 \times 3 \times 2$ factorial, with number of slide presentations (one, two), type of postevent information (misled-plus-commit, misled-only, control), and type of final test (standard, modified) all manipulated between subjects. The subjects ( $N=$ 216) were selected from the same sources as in Experiment 2, and 18 were assigned to each of the 12 conditions.

Procedure and Materials. The procedure and materials were the same as in Experiment 2 except that half of the subjects saw the slides once and half saw them twice. In the misled-plus-commit condition, misinformation was presented both in the narrative and on the interpolated test, whereas in the misled-only condition, misinformation was presented only in the narrative. Half of the subjects were assigned to each final test condition.

\section{Results and Discussion}

Table 1 shows the mean probabilities of correct recognition for all conditions. All three main effects were reliable sources of variation. Overall, recognition accuracy was higher following two slide presentations than following a single presentation $\left[F(1,204)=7.57, M S_{\mathrm{e}}=.07\right]$, and accuracy was lower on the standard test than on the 


\begin{tabular}{cccccc} 
& $\begin{array}{c}\text { Table 1 } \\
\text { Mean Probabilities of Correct Recognition as a Function of } \\
\text { Number of Slide Presentations, Final Testing Procedure, } \\
\text { and Type of Postevent Information (Experiment 3) }\end{array}$ \\
\hline $\begin{array}{ccccc}\text { No. Slide } \\
\text { Presentations }\end{array}$ & Type of Final Test & \multicolumn{3}{c}{ Type of Postevent Information } & \\
\hline One & Control & Misled-Only & Misled-Plus-Commit & $M$ \\
\hline Standard & .71 & .28 & .22 & .40 \\
Two & Modified & .72 & .71 & .58 & .67 \\
& Standard & .79 & .49 & .44 & .57 \\
& Modified & .76 & .67 & .65 & .69 \\
\hline
\end{tabular}

modified test $[F(1,204)=30.28]$. Type of postevent information also affected performance $[F(2,204)=21.66]$. Recognition accuracy was lowest in the misled-pluscommit condition, followed by the misled-only condition, with the control condition showing the highest level of accuracy overall. There also was an interaction between testing procedure and number of slide presentations $[F(1,204)=4.39]$. Although accuracy was lower on the standard test than on the modified test regardless of the number of slide presentations, the magnitude of the difference was larger following a single presentation (.40 and .67 for the standard and modified tests, respectively) than following two presentations (.57 and .69). As indicated in Table 1, the interaction between these factors was caused by variation in the misled conditions rather than by variation in the control condition. As such, this interaction indicates that the deleterious effect of presenting the misinformation as a response choice is greater the more weakly the original information is encoded. Presumably, the deleterious effect of increased access to the memory of the misinformation on the standard test is diminished when the original information is more accessible.

The interaction between type of postevent information and testing procedure also was reliable $[F(2,204)=8.15]$. The misinformation effect occurred both on the standard and the modified tests, but the effect was larger on the standard test. Pooled across number of slide presentations, the means on the standard test were $.33, .38$, and .75 , respectively, for the misled-plus-commit, misled-only, and control conditions. On the modified test, the corresponding means were $.62, .69$, and .74 . The difference in accuracy between the misled-only and the control conditions was significant on the standard test, but not on the modified test ( $L S D=.12)$, and this pattern replicates prior findings involving comparable conditions (e.g., those of McCloskey \& Zaragoza, 1985, and many others). Even more important, the difference between the misled-pluscommit and control condition was significant on both the standard and the modified tests. There were no other reliable sources of variation (all $F \mathrm{~s}<1$ ).

Although the findings indicated that the difference in accuracy between the misled-plus-commit and control conditions was greater in magnitude than the difference between the misled-only and the control conditions, other comparisons cloud the issue of whether commitment to misinformation always adds to the effect of read- ing about misinformation. The reason for this lack of clarity is that although accuracy was always lower in the misled-plus-commit condition than in the misled-only condition, sometimes substantially so (e.g., following a single slide presentation and given the modified test), the difference between these conditions in other cases was relatively small. One conclusion based on these results is that the effect of committing to misinformation does not always add much to the effect of reading about it. However, a different conclusion is more appropriate, based on comparing accuracy in the misled-plus-commit conditions from all three experiments with accuracy in misledonly conditions from many other studies that used the same materials we used.

We examined recognition accuracy from a subset of the studies Payne et al. (1994) included in their metaanalysis of misinformation effects on modified tests. Specifically, we pooled the means from all studies that used McCloskey and Zaragoza's (1985) stimuli and that compared performance in misled-only and control conditions ( $N=16$ pairs of means). The pooled means from these prior studies were identical to the corresponding means pooled across Experiments 1-3. In both cases, probability of correct recognition was .71 for the control condition and .69 for the misled-only condition. In contrast, recognition accuracy in the misled-plus-commit condition when pooled across Experiments 1-3 $(N=4)$ was only .55 , which is just a shade above chance. The appropriate conclusion based on this additional analysis is that reading about misinformation and then committing to it causes an additional decrement in performance on the modified test of about .14 compared with only reading about it. Other recent evidence using final recall tests confirms the assumption that active commitment has greater deleterious effects than passive reading (Schreiber \& Sergent, 1997).

The present findings also indicate that the misinformation effects that occurred were due to impaired memory in the misled-plus-commit condition rather than to a boost in performance in the control condition. Because we used control conditions in which people were presented with generic terms in the narrative and on the interpolated test (e.g., "tool"), it is conceivable that performance in the control condition could have been boosted if encoding such terms caused implicit rehearsal of the original information. However, there are three reasons 
why our findings cannot be explained in this way. First, implicit rehearsal of event information can also occur in the misled-plus-commit condition because the same generic terms were presented in that condition (e.g., when given the interpolated test question, "What tool did the man lift from his toolbox?"). Second, the means in our control conditions are similar to the means obtained in other studies. Third, and most important, accuracy on the modified test in the misled-plus-commit condition was most frequently near chance in all three experiments, which clearly indicates that memory was impaired. In contrast, accuracy in both the misled-only and the control conditions was equivalent and far above chance. This pattern cannot be explained by assuming that performance was enhanced in the control condition rather than impaired in the misled-plus-commit condition.

\section{GENERAL DISCUSSION}

All three experiments demonstrated that the misinformation effect occurs on modified recognition tests when people read about misinformation in a narrative and then selected it on an interpolated test. Like Belli (1993), we used McCloskey and Zaragoza's (1985) materials and very similar procedures. Unlike Belli, we used interpolated tests in which subjects could not choose the correct item. This procedure ensured that the effect of committing to correct information could not mask the effect of committing to misinformation. Our findings confirm those of Schooler et al. (1988), who also found the misinformation ef fect when using an interpolated test. Clearly, choosing incorrect information on an interpolated test impairs memory on a modified recognition test. The present findings also indicate that less interference is produced on a final test when subjects passively read about misinformation than when they read about it and then committed to it. Most often, accuracy on the final test in the misled-plus-commit condition was near chance. In Experiment 3, accuracy was always worse in the misled-plus-commit condition than in the misled-only condition, but the magnitude of the commitment effect was larger in some conditions than in others. Nevertheless, comparing the data from Experiments 1-3 with data from many other studies that used modified tests and the same materials we used supports the inference that reading about misinformation and then committing to it causes a greater decrement in performance than simply reading about it.

The most important finding of Experiment 3 was that accuracy on the modified test was lower in the misled-plus-commit condition than in the control condition, whereas accuracy in the misled-only condition was not significantly different from that in the control condition. To our knowledge, Experiment 3 is the first experiment investigating the misinformation effect that identifies a manipulation (the misled-plus-commit condition) that consistently produces substantial memory impairment on the modified test using McCloskey and Zaragoza's (1985) stimuli and that shows both impairment and no impairment (the misledonly condition) in the same experiment.

In terms of theoretical considerations, the present findings are compatible with the blocking hypothesis (Raaijmakers \& Shiffrin, 1981). This hypothesis predicted correctly that the misinformation effect would be larger in the misled-plus-commit condition than in the misledonly condition. It also predicted correctly that the misinformation effect would be larger on the standard test than on the modified test. Access to the misinformation should be more likely and the amount of interference should be greater when the misleading item is presented as a response choice than when it is not. The misleading item is an important cue for the memory of the misinformation. When this cue is available during the retrieval phase of the experiment, accessing the misinformation is more likely than when it is unavailable. The blocking hypothesis is also helpful for understanding why Belli (1993) did not obtain the misinformation effect. Prior retrieval of correct information should di- minish the deleterious effect of exposure to misinformation because commitment to correct information should block retrieval of misinformation.

The blocking hypothesis can also explain findings from studies using other versions of the repeated testing paradigm. Roediger et al. (1996) found that the extent to which performance was impaired on a final recall test depended on whether people reported misinformation or correct information on an interpolated recall test. Misinformation effects were reduced in conditions associated with higher rates of correct responding on the first test. These findings, too, are compatible with the blocking hypothesis. Once again, commitment to correct information on the first test insulates people against the deleterious effects of misinformation on a second test.

We have also found that reporting misinformation on an interpolated test impairs memory on a final recall test (Schreiber \& Sergent, 1997). The misinformation effect occurred on a final recall test even when people were warned immediately beforehand that misinformation had been presented, and it occurred even when people were told specifically that the misleading item "screwdriver" had been presented in the postevent materials and that this item was definitely incorrect. People understood these instructions; they never reported the misleading item. Nevertheless, recall of the original information was still lower in the misled condition than in the control condition. We believe that this finding, along with the present research, provides strong support for the blocking hypothesis. Telling people about the misleading item right before they complete the final test causes them to think about it. They cannot avoid thinking about the misleading item because the instructions informed them that it was definitely wrong and must not be reported. The blocking hypothesis would assume that thinking about the misinformation repeatedly when searching memory for the slides blocks retrieval of the correct information.

\section{REFERENCES}

Anderson, M. C., \& Spellman, B. A. (1995). On the status of inhibitory mechanisms in cognition: Memory retrieval as a model case. Psychological Review, 102, 68-100.

BELLI, R. F. (1993). Failures of interpolated tests in inducing memory impairment with final modified tests: Evidence unfavorable to the blocking hypothesis. American Journal of Psychology, 106, 407-427.

ChANDLER, C. C. (1991). How memory for an event is influenced by related events: Interference in modified recognition tests. Journal of Experimental Psychology: Learning, Memory, \& Cognition, 17, 1 15-125.

LofTUs, E. F. (1979). Eyewitness testimony. Cambridge, MA: Harvard University Press.

Loftus, E. F., Miller, D. G., \& Burns, H. J. (1978). Semantic integration of verbal information into a visual memory. Journal of Experimental Psychology: Human Learning \& Memory, 4, 19-31.

McCloskey, M., \& Zaragoza, M. S. (1985). Misleading postevent information and memory for events: Arguments and evidence against memory impairment hypotheses. Journal of Experimental Psychology: General, 114, 1-16.

MСGеосH, J. A. (1942). The psychology of human learning. New York: Longmans, Green.

Nelson, D. L., SChreiber, T. A., \& McEvoy, C. L. (1992). Processing implicit and explicit representations. Psychological Review, 99. 322-348.

Payne, D. G., Toglia, M. P., \& Anastasi, J. S. (1994). Recognition performance level and the magnitude of the misinformation effect in eyewitness memory. Psychonomic Bulletin \& Review, 1, 376-382.

RaAijmakers, J. G. W., \& Shiffrin, R. M. (1981). Search of associative memory. Psychological Review, 88, 93-134.

ROEDIGER, H. L., III, JACOBY, D., \& MCDERMOTt, X. (1996). Misinformation effects in recall: Creating false memories through repeated testing. Journal of Memory \& Language, 35, 300-318.

RoEdiGER, H. L., III, \& NeELY, J. H. (1982). Retrieval blocks in episodic and semantic memory. Canadian Journal of Psychology, 36, 213-242. SCHOOLER, J. W., Foster, R. A., \& Loftus, E. F. (1988). Some deleterious consequences of the act of recollection. Memory \& Cognition. 16. 243-251.

SChreiber, T. A., \& Sergent, S. D. (1997). Producing misinformation 
effects on standard and modified logic of opposition tests of eyewitness memory. Manuscript submitted for publication.

\section{NOTES}

1. Schooler et al. (1988) also discussed the practical implications of the commitment effect. Eyewitnesses may be asked to remember what they saw on multiple occasions. If they committed to erroneous information on one or more of those occasions, the commitment effect indicates that on subsequent occasions they will have difficulty remembering what they witnessed.
2. A related problem in Belli's (1993) experiment is that people in the control condition could give the misleading item or some other incorrect item as a response on the interpolated test. Whenever this occurred, control subjects were committing to misinformation. Committing to incorrect information in the control condition should lower accuracy on the final test and also should reduce the chances of finding the misinformation effect.

(Manuscript received September 22, 1997; revision accepted for publication December 23, 1997.) 